\title{
Real-world Experience With Sunitinib Treatment in Patients With Metastatic Renal Cell Carcinoma: Clinical Outcome According to Risk Score
}

\author{
Manuela Schmidinger, ${ }^{1}$ Camillo Porta, ${ }^{2}$ Stephane Oudard, ${ }^{3}$ Gwenael Denechere, ${ }^{4}$ \\ Yves Brault, ${ }^{5}$ Lucile Serfass, ${ }^{4}$ Nuno Costa, ${ }^{6}$ James Larkin ${ }^{7}$
}

\begin{abstract}
ADONIS is an ongoing observational study designed to evaluate treatment patterns/outcomes in patients with metastatic renal cell carcinoma treated with first-line sunitinib and/or second-line axitinib post sunitinib. We report an evaluation of sunitinib efficacy by risk group, in the real-world setting examined by ADONIS. Sunitinib efficacy was also assessed in intermediate-risk subgroups with 1 and 2 risk factors.

Background: ADONIS is an ongoing observational study in 9 European countries, designed to evaluate treatment patterns/outcomes in patients with metastatic renal cell carcinoma (mRCC) treated with first-line sunitinib and/or second-line axitinib post sunitinib. We present an evaluation of sunitinib efficacy by risk group, in the real-world setting examined in ADONIS. Patients and Methods: Patients were enrolled at the start of first-line sunitinib treatment or second-line axitinib post sunitinib treatment. Evaluation of sunitinib efficacy was assessed by International Metastatic Renal Cell Carcinoma Database Consortium (IMDC) and Memorial Sloan Kettering Cancer Center risk criteria. Results: For all patients in this analysis $(\mathrm{N}=467)$, the median progression-free survival was 23.8 months $(95 \%$ confidence interval [Cl], 16.5-28.5 months), 11.8 months ( $95 \% \mathrm{Cl}$, 8.1-17.4 months), and 4.6 months (95\% Cl, 2.5-7.7 months) for IMDC favorable-, intermediate-, and poor-risk groups, respectively. The median overall survival was 97.1 months (95\% $\mathrm{Cl}, 46.3$ months-not evaluable [NE]), 33.5 months ( $95 \% \mathrm{Cl}, 20.5-46.6$ months), and 10.0 months $(95 \% \mathrm{Cl}, 4.5-19.8$ months) for the respective risk groups. Data on individual risk factors were available for a subgroup of patients, allowing analysis by intermediate risk by 1 versus 2 risk factors. When including this subgroup $(n=120)$, the median overall survival for IMDC favorable-, intermediate-1, and intermediate-2 risk factors was 21.6 months $(95 \% \mathrm{Cl}$, 16.3 months-NE), 20.5 months (15.5 months-NE), and 15.1 months (4.1 months-NE), respectively. Conclusions: For patients overall and by risk-group stratification, survival estimates were aligned with previously published data. In patients with intermediate-1 risk, overall survival was very similar to patients with favorable risk. However, further exploration of outcome data from different sources is needed to confirm these observations.
\end{abstract}

Clinical Genitourinary Cancer, Vol. ш, No. m, - -92020 The Authors. Published by Elsevier Inc. This is an open access article under the CC BY-NC-ND license (http://creativecommons.org/licenses/by-nc-nd/4.0/).

Keywords: ADONIS trial, Intermediate-risk, Kidney cancer, Risk stratification, Targeted therapy

\section{Introduction}

More than 400,000 cases of kidney cancer are diagnosed worldwide each year, and approximately 175,000 deaths are

\footnotetext{
${ }^{1}$ Clinical Division of Oncology, Department of Medicine I, Medical University of

Vienna, Vienna, Austria

${ }^{2}$ Department of Internal Medicine, University of Pavia and I.R.C.C.S. Istituti Clinici Scientifici Maugeri, Pavia, Italy

${ }^{3}$ Medical Oncology Department, European Hospital Georges Pompidou, University

René Descartes, Paris, France

${ }^{4}$ Pfizer Oncology, Paris, France

${ }^{5}$ Pfizer, Paris, France

${ }^{6}$ Pfizer Biofarmaceutica, Lisbon, Portugal

${ }^{7}$ The Royal Marsden, NHS Foundation Trust, London, UK
}

attributed to the disease. The rate of kidney cancer is higher in developed counties versus less-developed countries, with approximately 115,000 new cases in Europe annually. ${ }^{1,2}$ The majority of

Submitted: Dec 11, 2019; Revised: Feb 19, 2020; Accepted: Feb 25, 2020

Address for correspondence: Manuela Schmidinger, MD, Clinical Division of Oncology, Department of Medicine I, Medical University of Vienna, Wahringer Guertel 18-20, 1090 Vienna, Austria

E-mail contact: manuela.schmidinger@meduniwien.ac.at 


\section{Real-world Experience With Sunitinib by Risk}

these cases, upwards of $90 \%$, are renal cell carcinoma (RCC). ${ }^{1,2}$ The ADONIS trial is an ongoing, prospective/retrospective, observational study initiated at 158 sites distributed among 9 European countries, and has been designed to evaluate treatment patterns and outcomes in patients with metastatic RCC (mRCC) treated with first-line sunitinib and/or second-line axitinib post sunitinib.

Sunitinib is an orally available, small molecule, tyrosine kinase inhibitor (TKI) of vascular endothelial growth factor receptor (VEGF) receptors 1-3, platelet-derived growth factor (PDGF) receptor- $\alpha$, PDGF receptor- $\beta$, and other receptor tyrosine kinases that promote angiogenesis. It has been approved globally for the treatment of advanced RCC and has been a standard of treatment for over 12 years. ${ }^{3,4}$ It is currently considered an option for use in firstline treatment strategies for patients with $\mathrm{mRCC}$ and favorable-risk in both the National Comprehensive Cancer Network (NCCN) and the European Society for Medical Oncology (ESMO) guidelines. ${ }^{4-7}$ Axitinib is also an orally available, small molecule TKI of VEGF receptors 1-3 with anti-angiogenesis activity, approved for second-line treatment of mRCC in the United States (US) and Europe since $2012 .{ }^{8}$ In April 2019, the combination of pembrolizumab and axitinib was approved by the US Food and Drug Administration (FDA) for the treatment of mRCC in the first-line setting; this was closely followed in May 2019 by FDA approval of the combination of avelumab and axitinib, in the same patient setting. ${ }^{9-12}$ In September and October of 2019, the combinations of pembrolizumab and axitinib, and avelumab and axitinib, were approved respectively for the treatment of $\mathrm{mRCC}$ in the first-line by the European Medicines Agency. ${ }^{13,14}$

Previously, the combination of checkpoint inhibitors nivolumab plus ipilimumab was approved in the United States and Europe for patients with mRCC who have not received previous systemic treatment and who have intermediate/poor-risk classification. This approval was based on improved outcomes in patients with intermediate/poor risk with nivolumab/ipilimumab versus sunitinib in the Checkmate 214 trial. $^{6}$ As a secondary endpoint, outcomes (progression-free survival [PFS], overall response rate [ORR], and overall survival $[\mathrm{OS}]$ ) were evaluated for favorable-risk patients. In this population, sunitinib and nivolumab/ipilimumab efficacy were not statistically different. ${ }^{6,15}$

The analysis presented here includes the evaluation of sunitinib efficacy by International Metastatic Renal Cell Carcinoma Database Consortium (IMDC) and Memorial Sloan Kettering Cancer Center (MSKCC) risk criteria. The IMDC prognostic model, first proposed and validated in 2009, uses 6 factors (diagnosis to treatment-time interval, Karnofsky performance status scale, hemoglobin level, platelet count, neutrophil count, and serum calcium concentration) in order to classify patients into favorable- ( 0 risk factors), intermediate- ( 1 or 2 risk factors), and poor-risk ( $\geq 3$ risk factors) prognostic groups. ${ }^{16}$

The MSKCC model, first proposed in 2002, uses 5 factors, 4 of which are shared with the IMDC model: time from diagnosis to treatment, Karnofsky performance status, hemoglobin, and serum calcium. The MSKCC model also includes serum lactate dehydrogenase concentration. The MSKCC model has also been used to stratify patients into favorable- ( 0 risk factors), intermediate- ( 1 or 2 risk factors), and poor-risk ( $\geq 3$ risk factors) groups. ${ }^{17}$

In the current study, an additional analysis of sunitinib efficacy was performed in a subset of patients identified as the risk factor set
(RFS), for whom individual risk factor data were available. This analysis was performed by IMDC intermediate-risk with 1 risk factor (intermediate-1) versus 2 risk factors (intermediate-2). The safety profile for sunitinib in real-world settings as observed in the ADONIS trial is also reported.

This study presents a real-world assessment of sunitinib efficacy and safety that confirms outcomes reported from the clinical trial setting. As sunitinib remains a first-line option for patients with favorable risk, along with the newly approved axitinib and checkpoint inhibitor combinations, a deeper understanding of their efficacy and safety, as demonstrated in the real-world setting, can help inform considerations of their use as an option in the treatment of mRCC going forward. ${ }^{6,12}$

Future analyses of the ADONIS study will include treatment sequencing data for sunitinib followed by axitinib as well as other treatments (cabozantinib, nivolumab, and others), sunitinib and axitinib therapy management strategies, and patient-reported quality of life measures.

\section{Patients and Methods \\ Patients and Study Design}

Patients were enrolled at the start of first-line treatment with sunitinib or second-line axitinib post sunitinib treatment. The full analysis set (FAS) includes all eligible patients enrolled in the study. The safety analysis set (SAS) includes all patients with at least 1 treatment intake documented (ie, date of first intake available), regardless of treatment (sunitinib or axitinib). For patients in the RFS, data on the 6 individual IMDC risk factors were available, allowing additional analysis by intermediate- 1 and intermediate- 2 risk factors.

The first patient was enrolled in this study on October 23, 2014. The data cutoff for this analysis was May 31, 2018. Analysis focused on first-line sunitinib in the metastatic setting. Data collection for the complete ADONIS trial is continuing, and as of May 1, 2019, 555 patients were enrolled.

\section{Key Eligibility Criteria}

Patients were $\geq 18$ years of age and had histologically confirmed diagnosis of mRCC (clear-cell or non-clear-cell renal tumor) with measurable disease according to Response Evaluation Criteria in Solid Tumors (RECIST) v1.1. Enrolled patients were treated with first-line sunitinib or second-line axitinib according to the approved therapeutic indication (except post cytokines) in Europe.

\section{Statistical Analysis}

Patients were grouped according to the IMDC and MSKCC prognostic models. For each prognostic model, patients were grouped into favorable-, intermediate-, or poor-risk groups. The RFS population had intermediate risk by IMDC and were additionally grouped according to the presence of 1 or 2 risk factors. Time-to-event endpoints of PFS and OS were estimated using Kaplan-Meier methods. Patients who discontinued the study for any reason or who were free of event at the cutoff date were censored at the time of their last disease assessment. Many patients in ADONIS are still under treatment, meaning that they did not have the events of interest. Two-sided log-rank tests were used to compare survival curves for sub-groups of interest. These 
comparisons were exploratory in nature. The type I error was set at $5 \%$. ORR was reported based on confirmed patient responses as assessed by treating clinicians.

\begin{tabular}{|c|c|c|c|}
\hline \multirow[t]{2}{*}{ Table 1} & \multirow[b]{2}{*}{$\begin{array}{c}\text { Prospective } \\
\text { Group (SU in } \\
\text { First-line at } \\
\text { Inclusion) } \\
\mathbf{n = 3 1 8} \\
\mathbf{n}(\%)\end{array}$} & \\
\hline & & $\begin{array}{l}\text { Retrospective/ } \\
\text { Prospective } \\
\text { Group (AXI in } \\
\text { Second-line at } \\
\text { Inclusion) } \\
\text { n = 149, } \\
\text { n (\%) }\end{array}$ & $\begin{array}{c}\text { Total N }=467 \\
\text { n (\%) }\end{array}$ \\
\hline \multicolumn{4}{|l|}{$\begin{array}{l}\text { Treatment at } \\
\text { inclusion }\end{array}$} \\
\hline $\mathrm{n}$ & 318 & 149 & 467 \\
\hline SU first-line & $318(100.0)$ & $0(0.0)$ & $318(68.1)$ \\
\hline AXI second-line & $0(0.0)$ & $149(100.0)$ & $149(31.9)$ \\
\hline \multicolumn{4}{|l|}{$\begin{array}{l}\text { Age at SU } \\
\text { initiation, y }\end{array}$} \\
\hline$n$ & 314 & 147 & 461 \\
\hline Missing & 4 & 2 & 6 \\
\hline Mean (SD) & $63.8(10.0)$ & $60.9(11.0)$ & $62.9(10.4)$ \\
\hline Median & 65 & 62 & 64 \\
\hline Min-max & $33-90$ & $31-88$ & $31-90$ \\
\hline Q1-Q3 & $57-71$ & $53-68$ & $56-70$ \\
\hline \multicolumn{4}{|l|}{$\begin{array}{l}\text { Age at SU initiation } \\
\text { category, y }\end{array}$} \\
\hline$n$ & 314 & 147 & 461 \\
\hline Missing & 4 & 2 & 6 \\
\hline$<65$ & $153(48.7)$ & 88 (59.9) & $241(52.3)$ \\
\hline$\geq 65$ & $161(51.3)$ & 59 (40.1) & $220(47.7)$ \\
\hline \multicolumn{4}{|l|}{ Gender } \\
\hline $\mathrm{n}$ & 318 & 149 & 467 \\
\hline Male & 237 (74.5) & $126(84.6)$ & $363(77.7)$ \\
\hline Female & 81 (25.5) & 23 (15.4) & $104(22.3)$ \\
\hline \multicolumn{4}{|l|}{$\begin{array}{l}\text { ECOG PS at SU } \\
\text { initiation }\end{array}$} \\
\hline$n$ & 260 & 108 & 368 \\
\hline Missing & 58 & 41 & 99 \\
\hline 0 & $123(47.3)$ & 57 (52.8) & $180(48.9)$ \\
\hline 1 & $114(43.8)$ & 38 (35.2) & $152(41.3)$ \\
\hline 2 & $21(8.1)$ & 11 (10.2) & $32(8.7)$ \\
\hline 3 & $2(0.8)$ & $2(1.9)$ & $4(1.1)$ \\
\hline
\end{tabular}

Abbreviations: $\mathrm{AXI}=$ axitinib; $\mathrm{ECOG} \mathrm{PS}=$ Eastern Cooperative Oncology Group performance status; $\mathrm{Q} 1$ = first quartile; $\mathrm{Q} 3=$ third quartile; $\mathrm{SD}=$ standard deviation; $\mathrm{SU}=$ sunitinib.

\section{Results \\ Patient Population}

At the time of data cutoff, there was a total of 467 patients in the FAS, with 462 patients in the SAS. Of the FAS group, there were 120 patients for whom complete individual risk data were available (ie, the RFS subgroup). Overall, patients in the study were a median age of 63 years (range, 31-90 years). The population was predominantly $(77.7 \%)$ male. At sunitinib initiation, 90.2\% of patients had Eastern Cooperative Oncology Group performance score $<2$ and $9.8 \%$ had Eastern Cooperative Oncology Group performance score $\geq 2$. The median follow-up time for patients treated with sunitinib in the first-line at inclusion was 9.2 months (range, 0.0-40.0 months) (Table 1). The majority $(81 \%)$ of patients were started on sunitinib $50 \mathrm{mg} /$ day on schedule $4 / 2$ with some patients started at either a lower dose (primarily 37.5 or $25 \mathrm{mg} /$ day) and/or an alternate schedule (primarily schedule 2/1).

\section{Outcomes}

Overall, patients in FAS treated with first-line sunitinib had a median PFS of 10.4 months (95\% confidence interval [CI], 9.3-12.5 months) and a median OS of 34.0 months (95\% CI, 28.3-46.6 months). PFS and OS by IMDC risk group are shown in Table 2, and the Kaplan-Meier plots for these results are shown in Figure 1. Kaplan-Meier PFS and OS plots by MSKCC risk group for FAS are shown in Figure 2, with results summarized in Table 3.

PFS and OS estimates by IMDC intermediate-1 risk factor versus intermediate- 2 risk factors calculated for the RFS subgroup of patients $(\mathrm{n}=120)$ are shown in Table 4, with corresponding Kaplan-Meier plots illustrated in Figure 3. PFS and OS estimates were also calculated for the RFS subgroup, with patients with favorable- and intermediate-1 risk factor grouped together versus patients with poor- and intermediate- 2 risk factors. These data are shown in Table 5, with corresponding Kaplan-Meier plots illustrated in Figure 4. Additionally, the analysis of the frequency of individual risk factors for the RFS subgroup confirmed the findings published by Sella et a ${ }^{18}$ with time from diagnosis to treatment $<1$ year $(65.8 \%)$ and low hemoglobin level $(43.3 \%)$ to be the 2 most frequent risk factors presented by patients with $\mathrm{mRCC}$ at diagnosis, as shown in Table 6.

ORR by MSKCC risk group are presented in Table 7 . Risk classification was not provided by investigators for 217 (46.8\%) patients. The response rate for sunitinib-treated patients without risk classification $(42.1 \%)$ was comparable with favorable-risk patients (41.8\%).

Table 2 PFS and OS for First-line Sunitinib-treated Patients, by IMDC Risk Group

\begin{tabular}{|c|c|c|c|}
\hline IMDC Risk Group n $=238^{\mathrm{a}}$ & $\begin{array}{c}\text { Favorable } \\
n=73(15.7 \%)\end{array}$ & Intermediate $n=117(25.2 \%)$ & $\begin{array}{c}\text { Poor } \\
n=48(10.3 \%)\end{array}$ \\
\hline Median PFS, mos (95\% Cl) & $23.8(16.5-28.5)$ & $11.8(8.1-17.4)$ & $4.6(2.5-7.7)$ \\
\hline Median OS, mos (95\% Cl) & $97.1(46.3-N E)^{b}$ & $33.5(20.5-46.6)$ & $10.0(4.5-19.8)$ \\
\hline
\end{tabular}

Abbreviations: $\mathrm{Cl}=$ confidence interval; IMDC = International Metastatic Renal Cell Carcinoma Database Consortium; NE = not evaluable; OS = overall survival; PFS = progression-free survival.

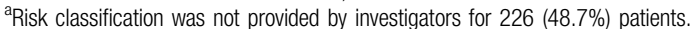

${ }^{b}$ Median OS assessed on a very small set of patients at risk $(n=3)$. Susceptible to change with a longer follow-up. 


\section{Real-world Experience With Sunitinib by Risk}

\section{Figure 1 Kaplan-Meier Estimate of PFS (A) and OS (B) for First-Line Sunitinib, by IMDC Risk Group. ${ }^{a}$ Risk Classification for 226 (48.7\%) Patients was Not provided by Investigators}

\section{A Progression-free survival}

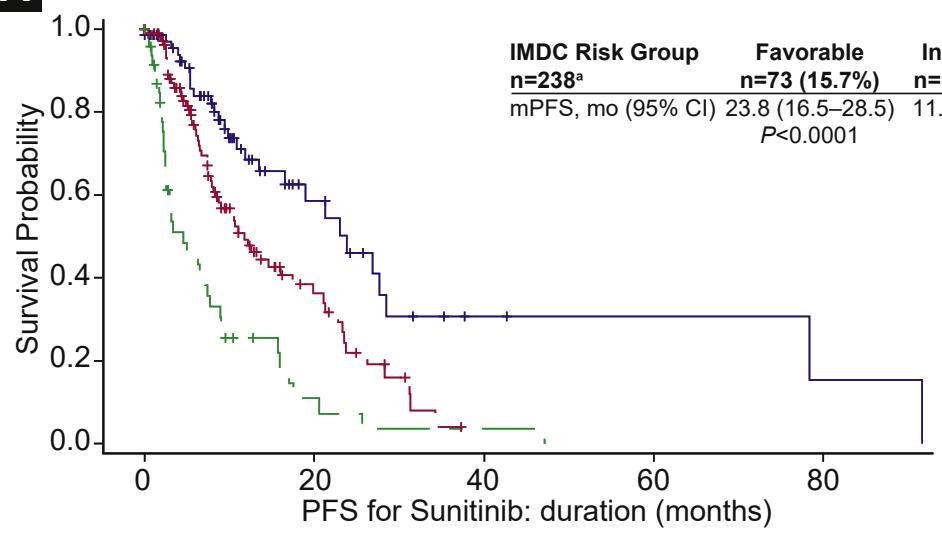

\section{B Overall survival}

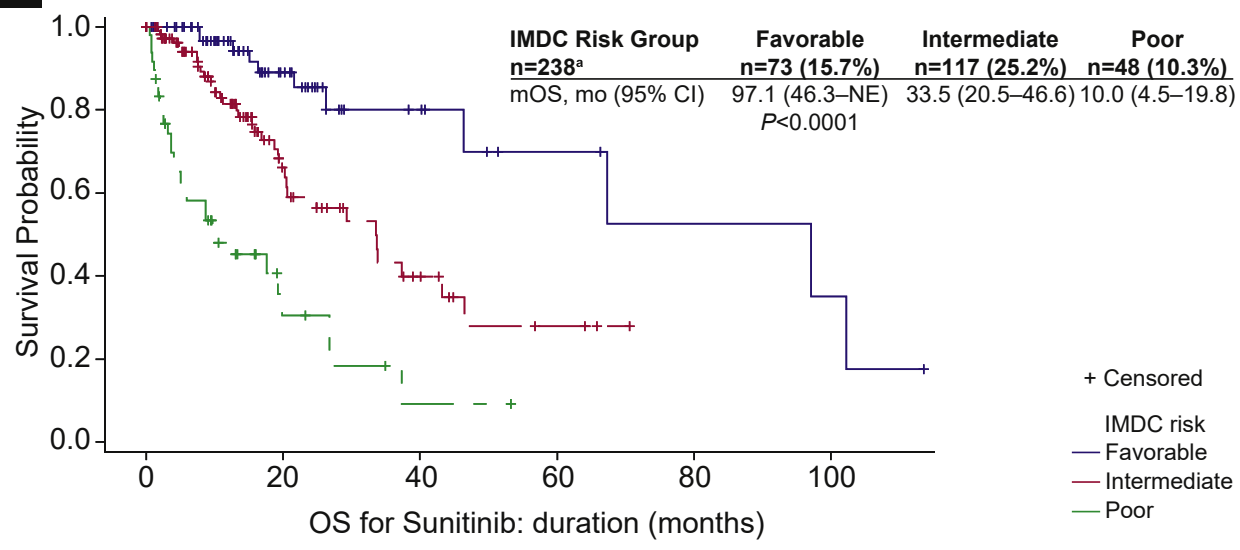

Abbreviations: $\mathrm{Cl}=$ confidence interval; IMDC = International Metastatic Renal Cell Carcinoma Database Consortium; mOS = median overall survival; mPFS = median progression-free survival; $\mathrm{NE}=$ not evaluable.

\section{Safety of Sunitinib}

Safety endpoints were evaluated in all 462 patients enrolled in this study that were treated at least once with either sunitinib or axitinib (SAS). Results were aligned with previous experience, and no new or unexpected safety events were observed so far.

\section{Discussion}

In the current NCCN and ESMO treatment guidelines, sunitinib, along with 2 other VEGF-targeted treatments, pazopanib and bevacizumab plus interferon, are listed as first-line treatment options for patients with mRCC and favorable risk. ${ }^{5,7,19}$ ESMO treatment guidelines also include the selective VEGF inhibitor tivozanib as an option in the first-line treatment of patients with favorable risk. ${ }^{5}$ Nivolumab/ipilimumab is currently the preferred first-line treatments for patients with intermediate-/poor-risk factors and also an option for patients with favorable risk, with cabozantinib as an option in both scenarios. $5,7,19$ Additionally, the combinations of pembrolizumab/axitinib and avelumab/axitinib were approved by the FDA in April 2019 and May 2019, respectively, for the treatment of patients with mRCC and any prognostic risk. ${ }^{9,11,20,21}$ The European Medicine Agency approved the pembrolizumab/ axitinib and avelumab/axitinib combinations for the treatment of mRCC in the first-line in September and October of 2019 respectively. ${ }^{13,14}$ Although sunitinib is not the newest treatment option for patients with intermediate-/poor-risk prognostic factors, it may still be considered for these patients if newer treatments are unavailable owing to local approval status, or in consideration of the greater clinical experience with the safety profile and efficacy of VEGF-targeted agents (ie, patients with features and comorbidities like compromised immune-system conditions will not be good candidates for immuno-oncology therapy). 5,9,11

As the mRCC treatment landscape continues to evolve, established VEGF-targeted agents, such as sunitinib, pazopanib, and tivozanib, are likely to continue to also have utility in second-line treatment after progression post nivolumab/ipilimumab, post axitinib/pembrolizumab, post axitinib/avelumab, or post cabozantinib treatment, as well as in potential combination therapy with immuno-oncology agents. There have been reports of efficacy for 

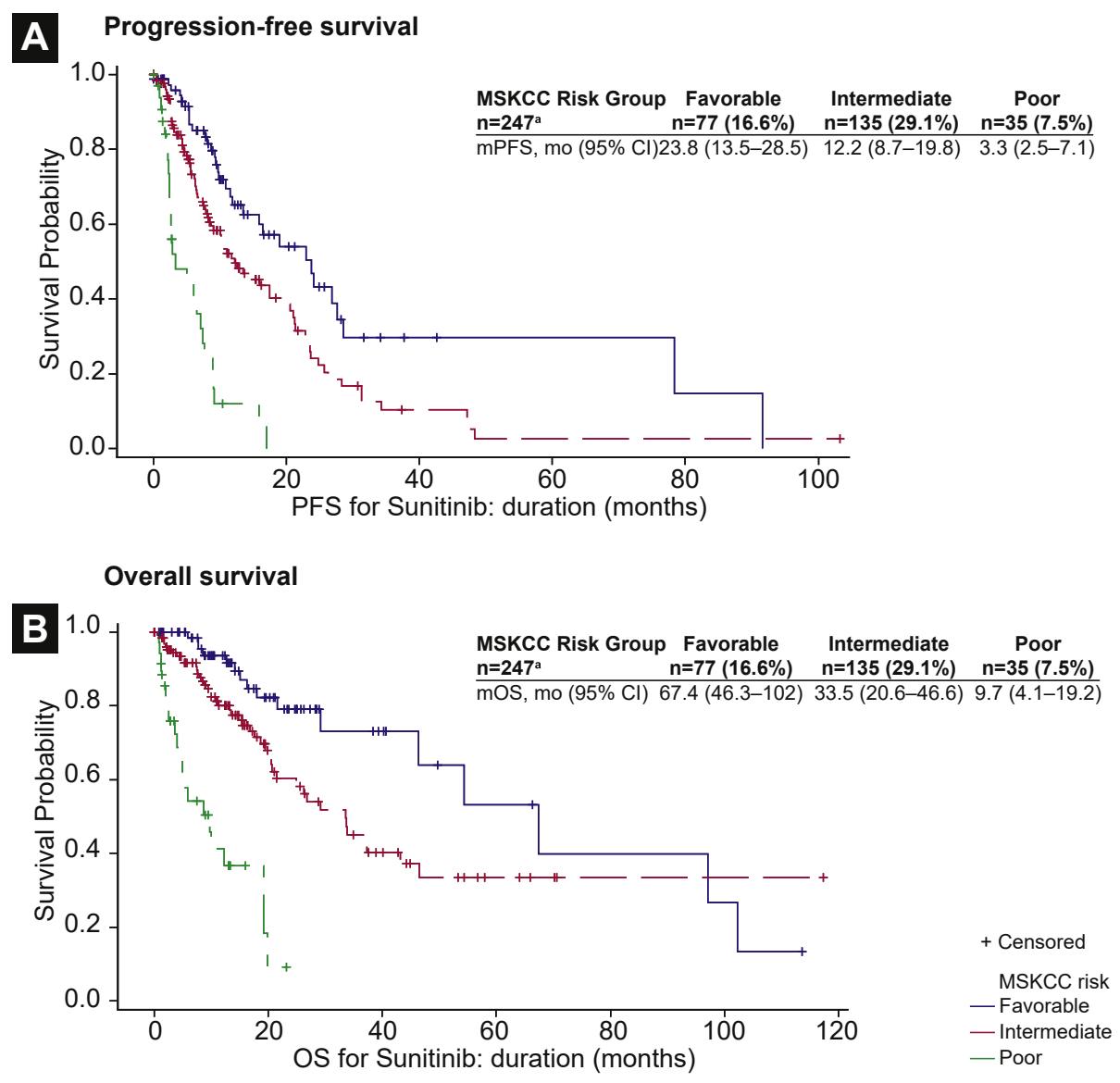

Abbreviations: $\mathrm{Cl}=$ confidence interval; $\mathrm{mOS}=$ median overall survival; $\mathrm{mPFS}=$ median progression-free survival; MSKCC = Memorial Sloan Kettering Cancer Center.

VEGF-targeted treatments in patients with mRCC who experience relapse post checkpoint inhibitor therapy. ${ }^{22-30}$

Aspects of the real-word efficacy data for sunitinib in first-line treatment of mRCC reported here reflect the gains in clinical experience in the treatment of mRCC with targeted therapies, specifically sunitinib, over the past decade (ie, managing adverse events and maintaining patients on treatment at optimal dose). For example, in the pivotal sunitinib phase III trial, PFS for patients with IMDC favorable risk was 16.0 months (95\% CI, 13.6-17.3 months), ${ }^{31}$ whereas the PFS for all patients with favorable risk in the current ADONIS study was 23.8 months (95\% CI, 16.5-28.5 months).
The survival data presented here add to the context for interpretation of the reported efficacy of sunitinib from other prospective clinical trials. ${ }^{3,31,32}$ CABOSUN, a randomized phase II trial that enrolled only patients with intermediate/poor risk according to IMDC criteria, reported an ORR of $12 \%$ and a median PFS of 5.6 months (95\% CI, 3.4-8.1 months) for patients with mRCC treated with sunitinib in the first line. These results are lower than the efficacy for patients in the FAS with IMDC intermediate/poor risk reported in ADONIS, with an ORR of $36 \%$ and a median PFS of 8.9 months (95\% CI, 7.4-12.2 months). ${ }^{33}$ Differences in patient population and study design may have contributed to the relatively shorter PFS in the sunitinib cohort in CABOSUN as compared

\section{Table 3 PFS and OS for First-line Sunitinib-treated Patients, by MSKCC Risk Group}

\begin{tabular}{l|c|c|c}
\hline MSKCC Risk Group $\mathbf{n}=\mathbf{2 4 7}^{\mathrm{a}}$ & Favorable $\mathbf{n}=\mathbf{7 7} \mathbf{( 1 6 . 6 \% )}$ & Intermediate $\mathbf{n}=\mathbf{1 3 5} \mathbf{( 2 9 . 1 \% )}$ & Poor $\mathbf{n}=\mathbf{3 5} \mathbf{( 7 . 5 \% )}$ \\
\hline Median PFS, mos $(95 \% \mathrm{Cl})$ & $23.8(13.5-28.5)$ & $12.2(8.7-19.8)$ & $3.3(2.5-7.1)$ \\
\hline Median OS, mos $(95 \% \mathrm{Cl})$ & $67.4(46.3-102)$ & $33.5(20.6-46.6)$ & $9.7(4.1-19.2)$ \\
\hline
\end{tabular}

Abbreviations: $\mathrm{Cl}=$ confidence interval; $\mathrm{MSKCC}=$ Memorial Sloan Kettering Cancer Center; NE = not evaluable; OS = overall survival; PFS $=$ progression-free survival. aRisk classification was not provided by investigators for 217 (46.7\%) patients. 


\section{Real-world Experience With Sunitinib by Risk}

\begin{tabular}{l|l|c|c|c} 
Table $\mathbf{4}$ & $\begin{array}{l}\text { PFS and OS for First-line Sunitinib-treated Patients, by IMDC Risk Group, Including Intermediate-1 Versus -2 Risk Factors at } \\
\text { Start }\end{array}$ \\
$\begin{array}{l}\text { Calculated IMDC } \\
\mathbf{n}=\mathbf{1 2 0}\end{array}$ & Favorable $\mathbf{n}=\mathbf{2 2}(\mathbf{1 8 . 3} \%)$ & INT-1 $\mathbf{n}=\mathbf{2 8} \mathbf{( 2 3 . 3 \% )}$ & INT-2 $\mathbf{n}=\mathbf{1 7} \mathbf{( 1 4 . 2 \% )}$ & Poor $\mathbf{n}=\mathbf{5 3} \mathbf{( 4 4 . 2 \% )}$ \\
Median PFS, mos $(95 \% \mathrm{Cl})$ & $16.5(8.7-18.9)^{\mathrm{a}}$ & $9.0(6.3-\mathrm{NE})^{\mathrm{b}}$ & $4.6(2.5-10.7)$ & $2.7(2.4-3.4)$ \\
\hline Median OS, mos $(95 \% \mathrm{Cl})$ & $21.6(16.3-\mathrm{NE})^{\mathrm{C}}$ & $20.5(15.5-\mathrm{NE})^{\mathrm{d}}$ & $15.1(4.1-\mathrm{NE})$ & $8.7(4.9-12.3)$ \\
\hline
\end{tabular}

Abbreviations: $\mathrm{Cl}=$ confidence interval; IMDC = International Metastatic Renal Cell Carcinoma Database Consortium; INT-1/-2 = intermediate risk with $1 / 2$ risk factors; NE = not evaluable; OS = overall survival; PFS = progression-free survival.

a Log-rank test $P$-value $<.001$ for the comparison of favorable patients versus all other groups.

bog-rank test $P$-value $=.1074$ for the comparison of INT-1 versus INT-2.

Cog-rank test $P$-value $<.001$ for the comparison of favorable patients versus all other groups.

dog-rank test $P$-value $=.0264$ for the comparison of INT-1 versus INT-2 patients.

with the real-world data presented here. However, the efficacy reported for the FAS population in ADONIS is similar to that reported for patients with IMDC intermediate/poor risk (ORR,
$31 \%)$ in a retrospective study of data from the pivotal sunitinib phase III trial. ${ }^{31}$ Also, the median PFS in the phase III trial was 10.6 months (95\% CI, 8.1-10.9 months) for patients with IMDC

\section{Figure 3 Kaplan-Meier Estimate of PFS (A) and OS (B) for First-Line Sunitinib, by IMDC Risk Group Individual Risk Factor}
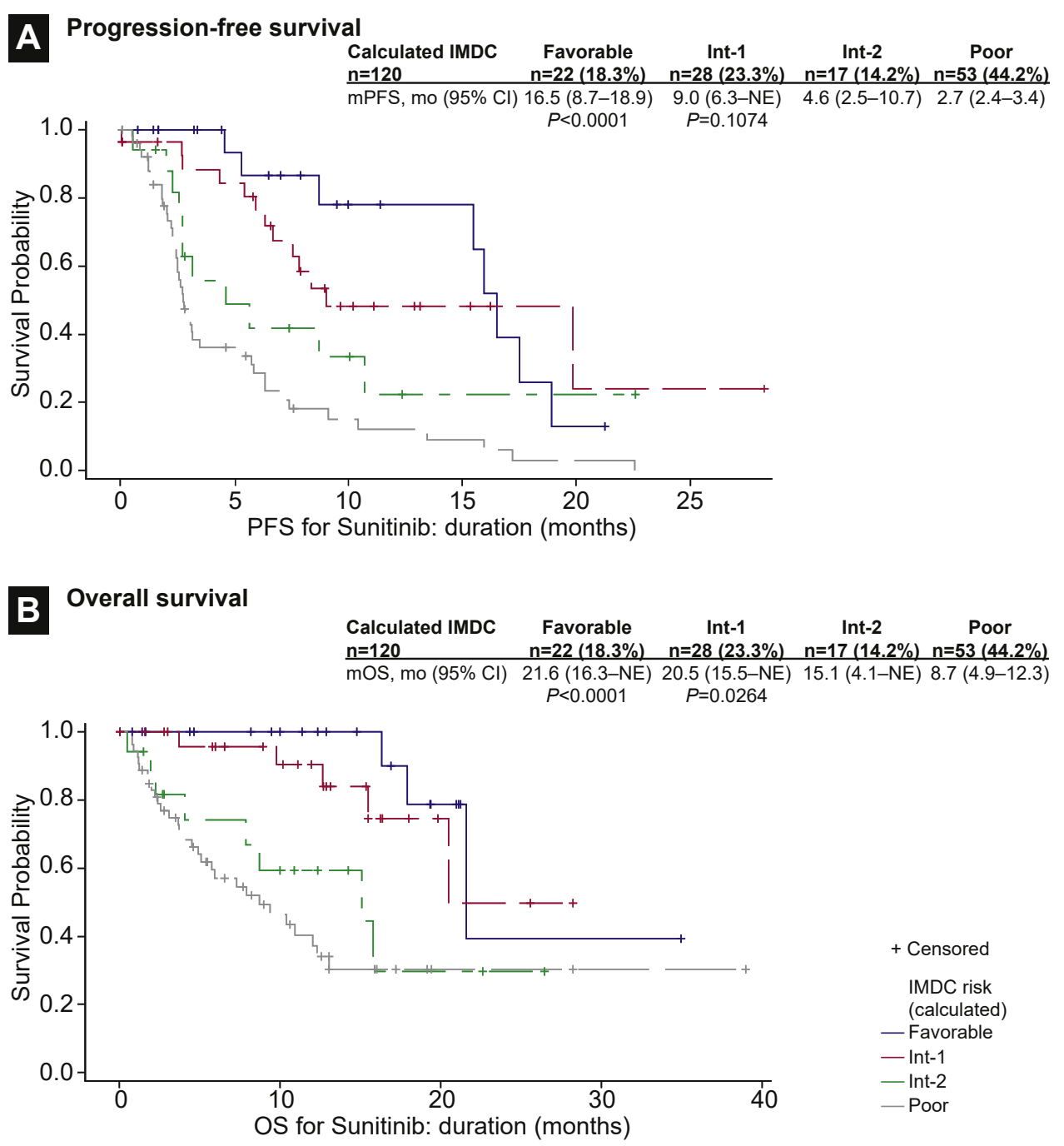

$$
\begin{gathered}
\text { + Censored } \\
\text { IMDC risk } \\
\text { (calculated) } \\
\text { — Favorable } \\
\text { — Int-1 } \\
\text { — Int-2 } \\
\text { — Poor }
\end{gathered}
$$

Abbreviations: $\mathrm{Cl}=$ confidence interval; IMDC = International Metastatic Renal Cell Carcinoma Database Consortium; Int- $1=1$ intermediate risk factor; Int- $2=2$ intermediate risk factors; m0S $=$ median overall survival; $\mathrm{mPFS}=$ median progression-free survival; NE $=$ not evaluable. 
Table 5 PFS and OS for First-line Sunitinib-treated Patients in the Risk Factor Set, by IMDC Risk Group, Favorable/ Intermediate-1 Versus Intermediate-2/Poor Risk Factors at Start

\begin{tabular}{|c|c|c|}
\hline $\begin{array}{l}\text { Calculated IMDC } \\
n=120\end{array}$ & $\begin{array}{l}\text { Favorable/INT-1 } \\
n=50(41.7 \%)\end{array}$ & $\begin{array}{c}\text { INT-2/Poor } \\
n=70(58.3 \%)\end{array}$ \\
\hline $\begin{array}{l}\text { Median PFS, mos } \\
(95 \% \mathrm{Cl})\end{array}$ & $15.9(8.3-18.9)^{a}$ & $2.8(2.5-5.1)$ \\
\hline $\begin{array}{l}\text { Median OS, mos } \\
(95 \% \mathrm{Cl})\end{array}$ & $21.6(20.5-\mathrm{NE})^{\mathrm{a}}$ & $9.4(5.7-15.1)$ \\
\hline
\end{tabular}

Abbreviations: $\mathrm{Cl}=$ confidence interval; IMDC = International Metastatic Renal Cell Carcinoma Database Consortium; INT-1/-2, intermediate risk with $1 / 2$ risk factors; NE = not evaluable; OS $=$ overall survival; PFS $=$ progression-free survival.

a Log-rank test $P$-value $<.001$ for the comparison of favorable/INT-1 versus INT-2/poor.

intermediate/poor risk; in other words, survival results in the phase III study were also longer than the PFS reported in CABOSUN. ${ }^{31}$

In the retrospective study of the aforementioned pivotal sunitinib phase III trial, the median PFS results in patients with intermediaterisk with 1-risk factor versus 2-risk factors were similar (10.9 months [95\% CI, 10.6-13.1 months] vs. 10.6 months [95\% CI, 7.1-13.7 months]). ${ }^{31}$ In contrast, the median PFS for patients with intermediate- 1 and intermediate- 2 risk in the RFS subgroup population were not similar ( 9.0 vs. 4.6 months, respectively). This difference is possibly owing to the subgroup of patients in the RFS subgroup not being fully representative of the FAS of the current study. ${ }^{31}$

The median OS in the pivotal sunitinib phase III trial was 28.2 months (95\% CI, 23.0 months-NE) and 16.3 months (95\% CI, 13.2-19.4 months) in patients with intermediate- 1 risk factor and intermediate- 2 risk factors, respectively. The current study also observed a difference between patients with 1 risk versus 2 risk factors in patients in the RFS subgroup. Moreover, patients in the RFS subgroup with favorable risk had a very similar median OS as patients with 1 risk factor. ${ }^{31}$

The finding reported here - that patients with intermediate-risk and 1 risk versus 2 risk factors may have different outcomes supports published analyses that suggest the intermediate-risk group, as defined by IMDC or MSKCC criteria, may be more

\section{Figure 4 Kaplan-Meier Estimate of PFS (A) and OS (B) for First-Line Sunitinib, by IMDC Risk Group Individual Risk Factors: Favorable/ Intermediate-1 Versus Intermediate-2/Poor}

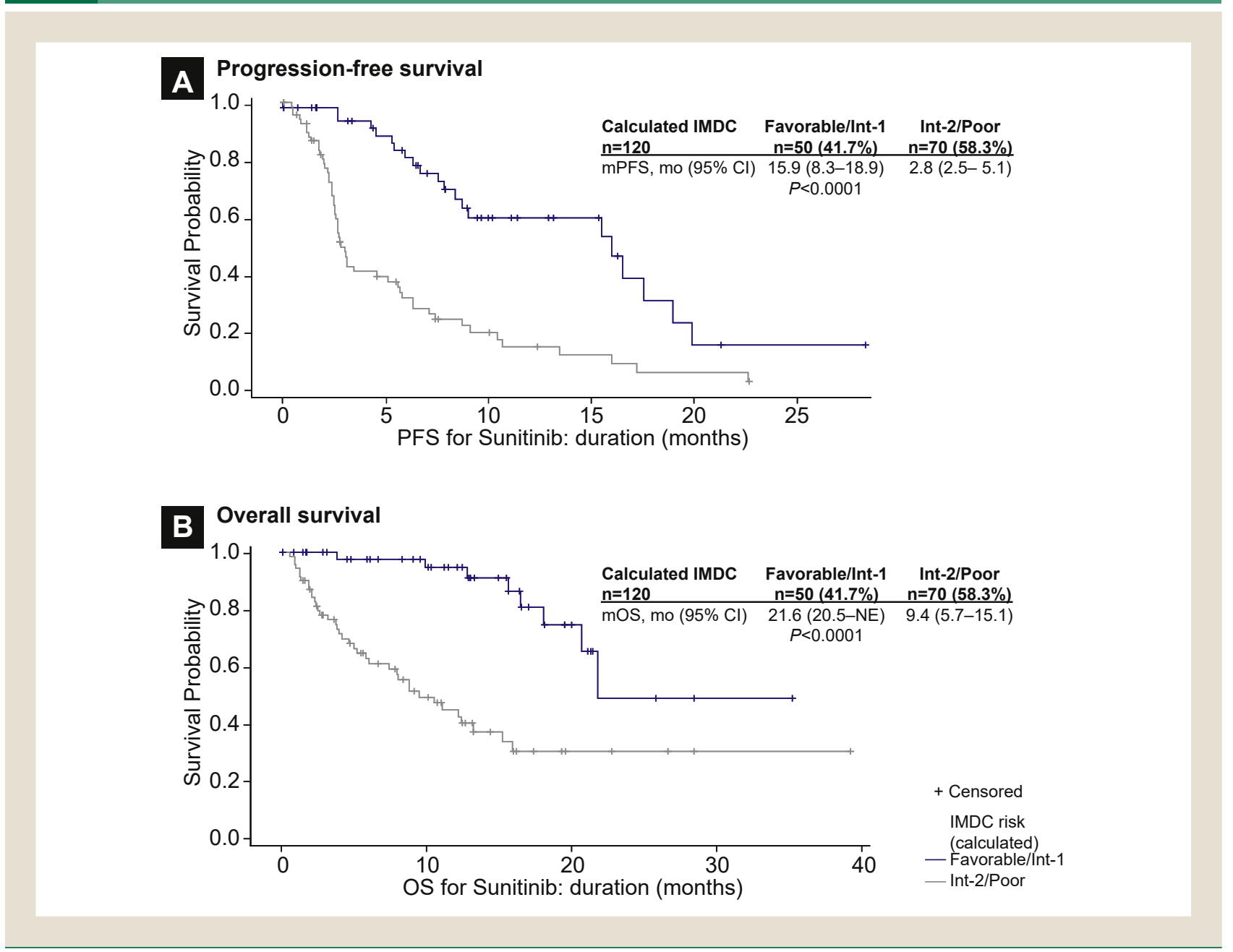

Abbreviations: $\mathrm{Cl}=$ confidence interval; IMDC = International Metastatic Renal Cell Carcinoma Database Consortium; Int- $1=1$ intermediate risk factor; Int- $2=2$ intermediate risk factors; $\mathrm{mOS}=$ median overall survival; $\mathrm{mPFS}=$ median progression-free survival; NE $=$ not evaluable. 


\section{Real-world Experience With Sunitinib by Risk}

Table 6 Frequency of Individual IMDC Risk Factors for Patients in the Risk Factor Set at Start

\begin{tabular}{l|c|c|c}
\hline IMDC Risk Factor & Factor Present, $\mathbf{n ~ ( \% ) ~}$ & Factor Absent, $\mathbf{n}(\mathbf{\%})$ & Data Missing \\
$<1$ year from diagnosis & $79(65.8)$ & $41(34.2)$ & - \\
\hline Hemoglobin < LLN & $52(43.3)$ & $68(56.7)$ & - \\
\hline Platelets $>$ ULN & $36(30.0)$ & $84(70.0)$ & - \\
\hline Neutrophils $>$ ULN & $32(27.1)$ & $86(72.9)$ & 2 \\
\hline Corrected calcium $>$ ULN & $31(26.5)$ & $86(73.5)$ & 3 \\
\hline KPS $<80 \%$ & $14(15.6)$ & $76(84.4)$ & 30 \\
\hline
\end{tabular}

Abbreviations: IMDC $=$ International Metastatic Renal Cell Carcinoma Database Consortium; KPS $=$ Karnofsky performance status; LLN $=$ lower limit of normal $\sim 12 \mathrm{~g} / \mathrm{dL} ; \mathrm{ULN}=$ upper limit of normal for platelets $\sim 150,000-400,000$ cells/ $\mu \mathrm{L} ; \mathrm{ULN}=$ upper limit of normal for neutrophils $\sim 2.0-7.0 \times 10^{9}$ cells/L; ULN = upper limit of normal for corrected calcium $\sim 8.5-10.2 \mathrm{mg} / \mathrm{dL}$. ${ }^{a} n=120$.

heterogeneous than patients with $\mathrm{mRCC}$ in the favorable- or poorrisk groups. ${ }^{18}$ Patients identified as intermediate risk have been the largest risk group entering treatment for mRCC in daily practice, with $20 \%$ diagnosed as having favorable risk, $45 \%$ with intermediate risk, and $35 \%$ with poor risk. ${ }^{34}$ Further exploration of the heterogeneity of this intermediate-risk group and the implications for use of prognostic tools are needed. ${ }^{35}$

This study has some limitations. One, it is limited by the nature of the retrospective post hoc component of the analysis. In addition, the RFS subgroup of patients was considerably smaller than the FAS population in this study, as the 6 individual risk factor data needed to calculate the IMDC risk score were only available for the smaller subset. As such, the RFS may not be fully representative of the larger FAS. Also, the OS data from this study may not represent the current and projected post disease-progression setting, owing to the emergence of immune-oncology therapies that have modified the treatment landscape. For example, the combination of pembrolizumab and axitinib demonstrated OS benefit over sunitinib in all IMDC patient risk groups in the phase III pivotal trial. ${ }^{12}$

\section{Conclusions}

For patients overall and by risk-group stratification, survival estimates for patients with mRCC treated with sunitinib in the ADONIS real-world study were aligned with results reported from the pivotal sunitinib phase III trial and with improvements seen in clinical practice over the past decade. The real-world characteristics of the study population support the soundness of these results. In patients with intermediate-risk with 1-risk factor, OS was very similar to patients with favorable-risk factors. However, further exploration is needed to confirm these observations.

\section{Clinical Practice Points}

- Real-word efficacy data from the ADONIS trial for sunitinib in first-line treatment of mRCC confirm the efficacy of sunitinib as reported in the pivotal phase III trial and reflect the gains in clinical experience over the last decade.

- Established VEGF-targeted agents, such as sunitinib, pazopanib, and tivozanib (only in Europe), are currently often used options for first-line treatment of $\mathrm{mRCC}$ for patients with favorable risk. For patients with intermediate- or poor-risk factors, newer treatments have been demonstrated to have survival benefits over targeted agents.

- The established targeted agents are likely to continue to have some utility in the first-line for patients with favorable risk.

- Targeted agents will also likely continue to have utility in secondline treatment after progression post nivolumab/ipilimumab, post axitinib/pembrolizumab, or post axitinib/avelumab, as well as in potential combination therapy with immuno-oncology agents.

\section{Data Sharing Statement}

Upon request, and subject to certain criteria, conditions, and exceptions (see https://www.pfizer.com/science/clinical-trials/trialdata-and-results for more information), Pfizer will provide access to individual de-identified participant data from Pfizer-sponsored global interventional clinical studies conducted for medicines, vaccines and medical devices (1) for indications that have been approved in the United States and/or European Union or (2) in programs that have been terminated (ie, development for all indications has been discontinued). Pfizer will also consider requests for the protocol, data dictionary, and statistical analysis plan. Data

\section{Table 7 Objective Response Rate for First-line Sunitinib-treated Patients, by MSKCC Risk Group}

\begin{tabular}{|c|c|c|c|c|c|}
\hline $\begin{array}{l}\text { MSKCC Risk Group } \\
\mathrm{n}=247^{\mathrm{a}}\end{array}$ & $\begin{array}{c}\text { Favorable } \\
\mathrm{n}=77(16.6 \%)\end{array}$ & $\begin{array}{c}\text { Intermediate } \\
\mathrm{n}=135(29.1 \%)\end{array}$ & Poor $n=35$ (7.5\%) & $\begin{array}{c}N A^{\mathrm{a}} \\
n=217(46.8 \%)\end{array}$ & Total $N=464$ \\
\hline \multicolumn{6}{|l|}{ ORR with sunitinib } \\
\hline$n$ & 67 & 107 & 23 & 183 & 380 \\
\hline Not reported & 10 & 28 & 12 & 34 & 84 \\
\hline No response, n (\%) & $39(58.2)$ & $68(63.6)$ & $15(65.2)$ & $106(57.9)$ & $228(60.0)$ \\
\hline Response, n (\%) & $28(41.8)$ & $39(36.4)$ & $8(34.8)$ & 77 (42.1) & $152(40.0)$ \\
\hline
\end{tabular}

Abbreviations: $\mathrm{Cl}=$ confidence interval; MSKCC $=$ Memorial Sloan Kettering Cancer Center; NA = not available; ORR = overall response rate ${ }^{a}$ Risk classification was not provided by investigators for 217 (46.8\%) patients. 
may be requested from Pfizer trials 24 months after study completion. The de-identified participant data will be made available to researchers whose proposals meet the research criteria and other conditions, and for which an exception does not apply, via a secure portal. To gain access, data requestors must enter into a data access agreement with Pfizer.

\section{CRediT authorship contribution statement}

Manuela Schmidinger: Conceptualization, Methodology, Formal analysis, Investigation, Resources, Writing - review \& editing, Supervision, Project administration, Visualization. Camillo Porta: Conceptualization, Methodology, Formal analysis, Investigation, Resources, Writing - review \& editing, Supervision. Stephane Oudard: Conceptualization, Methodology, Formal analysis, Investigation, Resources, Writing - review \& editing, Supervision. Gwenael Denechere: Conceptualization, Methodology, Formal analysis, Investigation, Resources, Data curation, Writing - review $\&$ editing, Supervision, Project administration, Visualization. Yves Brault: Conceptualization, Methodology, Formal analysis, Investigation, Resources, Data curation, Writing - review \& editing, Supervision. Lucile Serfass: Conceptualization, Methodology, Formal analysis, Investigation, Resources, Data curation, Writing - review $\&$ editing, Supervision, Project administration. Nuno Costa: Conceptualization, Methodology, Formal analysis, Investigation, Resources, Data curation, Writing - review \& editing, Supervision, Project administration. James Larkin: Conceptualization, Methodology, Formal analysis, Investigation, Resources, Writing - review $\&$ editing, Supervision, Project administration.

\section{Acknowledgments}

Medical writing support was provided by Charles Cheng, of Engage Scientific Solutions, and was funded by Pfizer Inc. This study was sponsored by Pfizer.

\section{Disclosure}

M Schmidinger has received speaker honoraria and consultancy fees from Pfizer Pharma GmbH, Roche, Bristol-Myers Squibb, MSD, Merck, Exelixis, Ipsen, Eisai, EUSA, Novartis, Alkermes, and Astellas. C Porta has received grants/research support from Pfizer, has served as a consultant for Novartis, Pfizer, BMS, Ipsen, Eisai, Janssen, EUSA, and Peloton; and has participated in speakers' bureaus for Novartis, Pfizer, BMS, Ipsen, and Eisai; S Oudard has received fees for board and scientific meetings from Pfizer, BMS, Bayer, Esai, Sanofi, Astellas, Janssen, MSD, and Novartis; J Larkin has participated in advisory board panels for Novartis, Pfizer, GSK, and Aveo Pharmaceuticals; and has received research funding from Novartis and Pfizer. G Denechere, Y Brault, L Serfass, and N Costa are employees of Pfizer and hold stock in Pfizer.

\section{References}

1. Ferlay J, Soerjomataram I, Dikshit R, et al. Cancer incidence and mortality worldwide: sources, methods and major patterns in GLOBOCAN 2012. Int J Cancer 2015; 136:E359-86.

2. Bray F, Ferlay J, Soerjomataram I, Siegel RL, Torre LA, Jemal A. Global cancer statistics 2018: GLOBOCAN estimates of incidence and mortality worldwide for 36 cancers in 185 countries. CA Cancer J Clin 2018; 68:394-424.
3. Motzer RJ, Hutson TE, Tomczak P, et al. Sunitinib versus interferon alfa in metastatic renal-cell carcinoma. N Engl J Med 2007; 356:115-24.

4. Pfizer Inc. Sutent (sunitinib) prescribing information. New York, NY, USA. Last update: May 2019, Available at: http://labeling.pfizer.com/ShowLabeling.aspx? $\mathrm{id}=607$. Accessed: October 25, 2019.

5. Escudier B, Grünwald V, Gillessen S, et al, ESMO Guidelines Committee. Renal cell carcinoma: ESMO Clinical Practice Guidelines for diagnosis, treatment and follow-up. Ann Oncol 2019; 30:706-20.

6. Motzer RJ, Tannir NM, McDermott DF, et al, CheckMate 214 Investigators Nivolumab plus ipilimumab versus sunitinib in advanced renal-cell carcinoma. N Engl J Med 2018; 378:1277-90.

7. Jonasch E. Updates to the management of kidney cancer. J Natl Compr Canc Netw 2018; 16:639-41.

8. Pfizer Inc. Inlyta (axitinib) prescribing information. New York, NY, USA: Pfizer. Last update: 2012, Available at: https://www.accessdata.fda.gov/drugsatfda_docs/ label/2012/202324lbl.pdf. Accessed: October 25, 2019.

9. Merck \& Co Inc. Keytruda (pembrolizumab) prescribing information. Whitehouse Station, NJ, USA: Merck \& Co., Inc. Last update: April 2019, Available at: https://www.accessdata.fda.gov/drugsatfda_docs/label/2019/125514Orig1s054lbl. pdf. Accessed: October 25, 2019.

10. Motzer RJ, Penkov K, Haanen J, et al. Avelumab plus axitinib versus sunitinib for advanced renal-cell carcinoma. N Engl J Med 2019; 380:1103-15.

11. EMD Serono Inc. Bavencio (avelumab) prescribing information. Rockland MA, USA: EMD Serono Inc. Last update: May 2019, Available at: https://www. emdserono.com/content/dam/web/corporate/non-images/country-specifics/us/pi/ bavencio-pi.pdf. Accessed: October 25, 2019.

12. Rini BI, Plimack ER, Stus V, et al, KEYNOTE-426 Investigators. Pembrolizumab plus axitinib versus sunitinib for advanced renal-cell carcinoma. $N$ Engl J Med 2019; 380:1116-27.

13. European Medicines Agency. Ketruda (pembrolizumab) summary of product characteristics. London: European Medicines Agency. Last update: September 11, 2019, Available at: https://www.ema.europa.eu/en/medicines/human/EPAR/ keytruda\#authorisation-details-section. Accessed: October 25, 2019.

14. Pfizer Inc. European Commission approves Bavencio ${ }^{\circledR}$ (avelumab) plus axitinib combination for first-line treatment of patients with advanced renal cell carcinoma. Darmstadt, Germany and New York, US. Last update: October 28, 2019, Available at: https:// www.pfizer.com/news/press-release/press-release-detail/european_commission_ approves_bavencio_avelumab_plus_axitinib_combination_for_first_line_ treatment_of_patients_with_advanced_renal_cell_carcinoma. Accessed: October 30, 2019 .

15. Bristol-Myers Squibb Co. Opdivo (nivolumab) prescribing information. Princeton, NJ, USA: Bristol-Myers Squibb. Last update: May 2019, Available at: https:// packageinserts.bms.com/pi/pi_opdivo.pdf. Accessed: October 25, 2019.

16. Heng DY, Xie W, Regan MM, et al. Prognostic factors for overall survival in patients with metastatic renal cell carcinoma treated with vascular endothelial growth factor-targeted agents: results from a large, multicenter study. J Clin Oncol 2009; 27:5794-9.

17. Motzer RJ, Bacik J, Murphy BA, Russo P, Mazumdar M. Interferon-alfa as a comparative treatment for clinical trials of new therapies against advanced renal cell carcinoma. J Clin Oncol 2002; 20:289-96.

18. Sella A, Michaelson MD, Matczak E, Simantov R, Lin X, Figlin RA. Heterogeneity of patients with intermediate-prognosis metastatic renal cell carcinoma treated with sunitinib. Clin Genitourin Cancer 2017; 15:291-9.e1.

19. Motzer RJ, Jonasch E, Michaelson MD, et al. NCCN Guidelines Insights: Kidney Cancer, Version 2.2020. I Natl Compr Canc Netw 2019; 17:1278-85.

20. Pfizer Inc. CHMP adoptes positive opinion for BAVENCIO (avelumab) plus axitinib for first-line treatment of patients with advanced renal cell carcinoma [media release]. Rockland, MD, and New York, NY: Pfizer. Last update: September 20, 2019, Available at: https://www.pfizer.com/news/press-release/ press-release-detail/chmp_adopts_positive_opinion_for_bavencio_avelumab_plus_ axitinib_for_first_line_treatment_of_patients_with_advanced_renal_cell_carcinoma. Accessed: October 25, 2019

21. BusinessWire. European Commission approves Merck's KEYTRUDA® (pembrolizumab) in combination with Inlyta ${ }^{\circledR}$ (axitinib) as first-line treatment for patients with advanced renal cell carcinoma (RCC) [media release]. Kenilworth, NJ, USA. Last update: September 4, 2019, Available at: https://www.businesswire. $\mathrm{com} /$ news/home/20190904005168/en/. Accessed: October 25, 2019.

22. Shah AY, Kotecha RR, Lemke EA, et al. Outcomes of patients with metastatic clear-cell renal cell carcinoma treated with second-line VEGFR-TKI after first-line immune checkpoint inhibitors. Eur J Cancer 2019; 114:67-75.

23. Auvray M, Auclin E, Barthelemy P, et al. Second-line targeted therapies after nivolumab-ipilimumab failure in metastatic renal cell carcinoma. Eur J Cancer 2019; 108:33-40.

24. Albiges L, Fay AP, Xie W, et al. Efficacy of targeted therapies after PD-1/ PD-L1 blockade in metastatic renal cell carcinoma. Eur J Cancer 2015; 51:2580-6.

25. Nadal R, Amin A, Geynisman DM, et al. Safety and clinical activity of vascular endothelial growth factor receptor (VEGFR)-tyrosine kinase inhibitors after programmed cell death 1 inhibitor treatment in patients with metastatic clear cell renal cell carcinoma. Ann Oncol 2016; 27:1304-11.

26. Barata PC, LA G, Mendirtta P, et al. Clinical outcome of patients (Pts) with metastatic renal cell carcinoma (mRCC) progressing on front-line immuneoncology based combination (IO-COMBO) regimens. J Clin Oncol 2018; $36(6$ Suppl): 613 


\section{Real-world Experience With Sunitinib by Risk}

27. Choueiri T, Motzer R, Campbell M, et al. Subgroup analysis from JAVELIN Renal 101: outcomes for avelumab plus axitinib $(A+A x)$ versus sunitinib $(S)$ in advanced renal cell carcinoma (aRCC). Presented at: American Society of Clinical Oncology (ASCO) Genitourinary Cancers Symposium, San Franciso, CA, USA, February 14-16, 2019. J Clin Oncol 2019; $37(7$ Suppl):544.

28. Powles T, Plimack E, Stus V, et al. Pembrolizumab (pembro) plus axitinib (axi) versus sunitinib as first-line therapy for locally advanced or metastatic renal cell carcinoma (mRCC): phase III KEYNOTE-426 study. Presented at: American Society of Clinical Oncology (ASCO) Genitourinary Cancers Symposium, San Francisco, CA, USA, February 14-16, 2019. J Clin Oncol 2019; $37(7$ Suppl):543.

29. Graham P, Wells JC, McKay R, et al. Clinical outcomes of patients with metastatic renal cell carcinoma (mRCC) treated with vascular endothelial growth factor receptor (VEGFR) tyrosine kinase inhibitors (TKI) and mammalian target of rapamycin inhibitors (mTORI) after immuno-oncology (IO) checkpoint inhibitors. Presented at: European Society of Medical Oncology (ESMO), Munich, Germany, October 19-23, 2018. Ann Oncol 2018; 29(Suppl 8):viii303-31.

30. Ornstein MC, Pal SK, Wood LS, et al. Individualised axitinib regimen for patients with metastatic renal cell carcinoma after treatment with checkpoint inhibitors: a multicentre, single-arm, phase 2 study. Lancet Oncol 2019; 20:1386-94.
31. Rini BI, Hutson TE, Figlin RA, et al. Sunitinib in patients with metastatic renal cell carcinoma: clinical outcome according to International Metastatic Renal Cell Carcinoma Database Consortium Risk Group. Clin Genitourin Cancer 2018; 16: 298-304.

32. Motzer RJ, Hutson TE, Tomczak P, et al. Overall survival and updated results for sunitinib compared with interferon alfa in patients with metastatic renal cell carcinoma. J Clin Oncol 2009; 27:3584-90.

33. Choueiri TK, Halabi S, Sanford BL, et al. Cabozantinib versus sunitinib as initial targeted therapy for patients with metastatic renal cell carcinoma of poor or intermediate risk: the Alliance A031203 CABOSUN Trial. J Clin Oncol 2017; 35: 591-7.

34. Iacovelli R, De Giorgi U, Galli L, et al. Is it possible to improve prognostic classification in patients affected by metastatic renal cell carcinoma with an intermediate or poor prognosis? Clin Genitourin Cancer 2018; 16:355-9.e1.

35. Escudier B, Tannir NM, McDermott DF, et al. CheckMate 214: efficacy and safety of nivolumab + ipilimumab $(\mathrm{N}+\mathrm{I})$ v sunitinib $(\mathrm{S})$ for treatment-naïve advanced or metastatic renal cell carcinoma (mRCC), including IMDC risk and PD-L1 expression subgroups. Presented at: 2017 Congress of the European Society for Medical Onoclogy (ESMO), Madrid, Spain, September 10th, 2017. Ann Oncol 2017; 28(Suppl 5):LBA5. 